\title{
Agreement between diagnoses of childhood lymphoma assigned in Uganda and by an international reference laboratory
}

This article was published in the following Dove Press journal:

Clinical Epidemiology

13 December 2012

Number of times this article has been viewed

\author{
Jackson Orem ${ }^{1-3}$ \\ Sven Sandin' \\ Caroline E Weibull' \\ Michael Odida ${ }^{4}$ \\ Henry Wabinga ${ }^{4}$ \\ Edward Mbidde ${ }^{2,3}$ \\ Fred Wabwire-Mangen ${ }^{5}$ \\ Chris JLM Meijer ${ }^{6}$ \\ Jaap M Middeldorp ${ }^{6}$ \\ Elisabete Weiderpass ${ }^{1,7,8}$ \\ 'Department of Medical Epidemiology \\ and Biostatistics, Karolinska Institutet, \\ Stockholm, Sweden; ${ }^{2}$ Uganda Cancer \\ Institute, ${ }^{3}$ School of Medicine, ${ }^{4}$ School \\ of Biomedical Sciences, ${ }^{5}$ School of \\ Public Health, Makerere University \\ College of Health Sciences, Kampala, \\ Uganda; ${ }^{6}$ Department of Pathology, \\ VU University Medical Center, \\ Amsterdam, The Netherlands; \\ ${ }^{7}$ Cancer Registry of Norway, Oslo; \\ Department of Community Medicine, \\ University of Tromsø, Tromsø, \\ Norway; ${ }^{8}$ Samfundet Folkhälsan, \\ Helsinki, Finland
}

Correspondence: Elisabete Weiderpass Department of Medical Epidemiology and Biostatistics, Karolinska Institutet, PO Box 28I, 17177 Stockholm, Sweden Tel +358408453406

$\mathrm{Fax}+4683$ I I I0।

Email elisabete.weiderpass@ki.se
Background: Correct diagnosis is key to appropriate treatment of cancer in children. However, diagnostic challenges are common in low-income and middle-income countries. The objective of the present study was to assess the agreement between a clinical diagnosis of childhood nonHodgkin lymphoma (NHL) assigned in Uganda, a pathological diagnosis assigned in Uganda, and a pathological diagnosis assigned in The Netherlands.

Methods: The study included children with suspected NHL referred to the Mulago National Referral Hospital, Kampala, Uganda, between 2004 and 2008. A clinical diagnosis was assigned at the Mulago National Referral Hospital, where tissue samples were also obtained. Hematoxylin and eosin-stained slides were used for histological diagnosis in Uganda, and were re-examined in a pathology laboratory in The Netherlands, where additional pathological, virological and serological testing was also carried out. Agreement between diagnostic sites was compared using kappa statistics.

Results: Clinical and pathological diagnoses from Uganda and pathological diagnosis from The Netherlands was available for 118 children. The agreement between clinical and pathological diagnoses of NHL assigned in Uganda was 91\% (95\% confidence interval [CI] 84-95; kappa $0.84 ; P<0.001)$ and in The Netherlands was $49 \%(95 \%$ CI $40-59$; kappa $0.04 ; P=0.612)$. When Burkitt's lymphoma was considered separately from other NHL, the agreement between clinical diagnoses in Uganda and pathological diagnoses in Uganda was 69\% (95\% CI 59-77; kappa $0.56 ; P<0.0001)$, and the corresponding agreement between pathological diagnoses assigned in The Netherlands was $32 \%(95 \%$ CI $24-41$; kappa $0.05 ; P=0.326)$. The agreement between all pathological diagnoses assigned in Uganda and The Netherlands was $36 \%(95 \%$ CI 28-46; kappa $0.11 ; P=0.046$ ).

Conclusion: Clinical diagnosis of NHL in Uganda has a high probability of error compared with pathological diagnosis in Uganda and in The Netherlands. In addition, agreement on the pathological diagnosis of NHL between Uganda and The Netherlands is very low.

Keywords: Africa, Epstein-Barr virus, non-Hodgkin lymphoma, Burkitt's lymphoma, cancer

\section{Introduction}

Correct diagnosis is key to appropriate treatment of cancer in general, and particularly in children, given that most childhood cancers are treatable and curable. ${ }^{1-3}$ However, diagnostic challenges are common in low-income and middle-income countries such as Uganda, where pathological diagnosis is still based on accurate determination of tissue architecture by histopathology. ${ }^{4,5}$ Conversely, additional laboratory tests, such as immunohistochemistry and flow cytometry, have become routine in pathology laboratories in high-income countries, and allow for more detailed classification, especially of hematological malignancies. 
Although there are few subtypes of childhood nonHodgkin lymphoma (NHL), ie, lymphoblastic lymphoma, Burkitt's or Burkitt's-like lymphoma, and large cell lymphoma, classification is usually challenging, ${ }^{6,7}$ and obtaining adequate tissue samples for histological or cytological diagnosis can be particularly difficult. ${ }^{2}$ Improper processing of materials, such as tissue handling and fixation, is the most common hindrance to a correct diagnosis in low-resource settings. ${ }^{2}$ The accuracy of diagnoses is therefore often in doubt, and disagreement between pathologists is common. ${ }^{8}$ Studies on the agreement between clinical and pathological diagnoses in Uganda and other low-income countries have not been carried out comprehensively, and the available information mainly pertains to adults. ${ }^{9,10}$

The assignment of appropriate treatment for cancer patients in sub-Saharan Africa is problematic, particularly for children. ${ }^{9,11}$ Indeed, appropriate treatment requires correct diagnosis, and in Uganda pathological diagnosis can be expensive, time-consuming, and even when available, results may be inconclusive for several reasons, including lack of adequate equipment and supplies to perform the necessary tests. ${ }^{9}$ However, even in the absence of a conclusive pathological diagnosis, clinicians must make decisions regarding treatment. As a result, initial patient management is determined by a combination of clinical, and, when available, pathological criteria. ${ }^{10,11}$ Moreover, in most countries in Africa, where childhood NHL is relatively common, once the treatment process is initiated, no further diagnostic refinement is done, mainly due to a lack of expertise, lack of appropriate laboratory facilities, and the high cost or unavailability of advanced tests. ${ }^{9}$ Therefore, it is difficult to ascertain the extent to which children are being treated for NHL without a definitive pathological diagnosis in these settings.

The objective of this study was to assess the agreement between clinical diagnoses of childhood NHL assigned in Uganda, pathological diagnoses assigned in Uganda, and pathological diagnoses assigned in a reference laboratory in The Netherlands. Our foremost motivations to perform this study was to elucidate whether childhood NHL in Uganda is underdiagnosed or overdiagnosed using current diagnostic practices and to ascertain the need to introduce more advanced diagnostic methods in the country.

\section{Materials and methods}

We used data from a case-control study of childhood NHL conducted between 2004 and 2008 at the Mulago National Referral Hospital (MNRH), Kampala, Uganda. Children with suspected childhood tumors were referred to the MNRH for evaluation, where they underwent clinical examination and were invited to participate in the study. Detailed clinical and demographic information was collected by questionnaire. All children received a clinical diagnosis at the MNRH, based on information on presentation and physical findings on clinical examination.

Children with suspected NHL were referred to the Uganda Cancer Institute in Kampala for specialized management. If the clinical diagnosis of NHL was confirmed at the Uganda Cancer Institute, the patient was considered a suspected case of NHL. Clinical diagnosis of Burkitt's lymphoma was defined as a diagnosis of NHL with a clinically identifiable facial tumor, which is usually typical of Burkitt's lymphoma. It was assumed that children with a clinical diagnosis of NHL without information on a facial tumor at clinical examination did not have a clinical diagnosis of Burkitt's lymphoma.

The cancer referral system in Uganda has been previously described in detail. ${ }^{12}$ Fresh tissue samples were collected at the Uganda Cancer Institute from all children with a suspected clinical diagnosis of NHL. All tissue samples were divided into two specimens; one was stored at $-80^{\circ} \mathrm{C}$ and the other was submitted to the Department of Pathology, Makerere University College of Health Sciences, Kampala, Uganda, for diagnosis according to a standardized protocol for paraffin-embedded tissue. ${ }^{13,14}$ Tissue samples were then examined morphologically and by hematoxylin and eosin staining in Uganda. Because pathology in Uganda is done as part of standard hospital procedure, the pathologists were not blinded to information on clinical diagnosis.

Biopsy blocks, fresh-frozen tissue samples, and original pathological slides that were judged to be of sufficient quality and quantity were then submitted for review to the Department of Pathology at the VU University Medical Center, Amsterdam, The Netherlands, which is an academic center of excellence for cancer pathology in Europe, and highly specialized in Epstein-Barr and other virus-related cancers. Morphological examinations were done in The Netherlands on the submitted slides by pathologists with expertise in lymphoma. These examinations were performed in a blinded manner, with the pathologists in The Netherlands having no information about the patients' clinical or pathological diagnoses from Uganda. Reporting criteria was based on presence of NHL (ie, Burkitt's lymphoma or other NHL), presence of other tumor, or presence of an inflammatory process with no tumor. New histological slides were then made from all tissue blocks received from Uganda, ie, 5-10 separate parallel sections were cut for hematoxylin and eosin staining, 
Epstein-Barr virus-encoded messenger RNA and RNA in situ hybridization (EBER-RISH) plus CD10, bcl2 and control stainings, including pan-B-cell antigen (CD19), pan-Tcell antigen (CD3), and cytokeratin-18, with all analyses performed using standardized procedures. Tissue samples were then subjected to further analysis with EBER-RISH, DAKO-PNA probes, and an optimized inhouse detection method. ${ }^{15}$

Results from both pathological laboratories (Uganda and The Netherlands) were classified as NHL (ie, Burkitt's lymphoma or other NHL), other cancers, and chronic noncancerous conditions. Subtype classification of NHL was also performed, and was categorized as Burkitt's lymphoma or other NHL. NHL diagnoses and subtyping were in accordance with the World Health Organization classification of hematological malignancies. ${ }^{?}$

\section{Statistical methods}

Agreement between clinical diagnoses at the MNRH, pathological diagnoses assigned in Uganda, and pathological diagnoses assigned in The Netherlands was established using summary statistics and by calculating kappa statistics with two-sided 95\% confidence intervals (CIs) using bootstrap techniques and a two-sided test of the hypothesis of a kappa being equal to zero. We calculated the observed agreement (percentage of samples rated equally) and two-sided 95\% exact CIs. ${ }^{16}$ The kappa statistic measures the agreement between two raters by relating the observed proportion of equally rated samples with what is expected from chance alone, ie, $\left(\mathrm{P}_{\mathrm{o}}-\mathrm{P}_{\mathrm{e}}\right) /\left(1-\mathrm{P}_{\mathrm{e}}\right)$, where $\mathrm{P}_{\mathrm{o}}$ is the observed proportion of samples on which two raters agree and $\mathrm{P}_{\mathrm{e}}$ is the proportion expected by chance alone. A value close to zero indicates no agreement while a value close to one indicates good agreement. ${ }^{17}$ The statistical software $\mathrm{R}$ package irr version 0.83 for Debian-64 bit was used for the kappa calculations.

\section{Results}

Between 2004 and 2008, 649 children aged 1-17 years with suspected tumors were referred to the MNRH for evaluation. Of these, 421 children who had their parents or guardians present were invited to participate in the study on childhood NHL. Assent was also requested from invited children aged 9 years or older. Assent was not requested from children aged younger than 9 years, because they were considered to be too young to understand the procedures; for these children, parental or guardian consent was considered sufficient for participation. Three hundred and twenty-one children had consent from a parent or guardian (and if aged 9 or more years assented to participate), and were thus enrolled. Of these 321 children, 314 had a clinical diagnosis of either a tumor or a nontumor condition (clinical diagnosis was missing for seven children). Tissue samples were collected from 273 enrolled children for pathological diagnosis in the Ugandan laboratory. Two hundred and thirty-nine of the tissue samples used for pathological diagnosis in Uganda were judged to be of sufficient quality and quantity and were shipped to The Netherlands for confirmatory diagnosis. Upon arrival in The Netherlands, 106 (44.4\%) of the samples were deemed not analyzable because they were either inadequate or poorly processed. Of the remaining 133 tissue samples, 129 could be conclusively diagnosed. There were 118 children with complete diagnostic information (clinical diagnosis, pathological diagnosis assigned in Uganda, and pathological diagnosis assigned in The Netherlands), among whom comparisons could be made at all levels (Figure 1).

\section{Patient characteristics}

Of the 314 children with a clinical diagnosis, 199 (63.4\%) were boys, 115 (36.6\%) were girls, and 13 children were positive for human immunodeficiency virus. The most common symptoms were fever, night sweats, and weight loss. The characteristics of the 118 children with complete diagnostic information were very similar to those of the 314 children with a clinical diagnosis (Table 1). The mean ages of these 314 and 118 children were $7.2 \pm 3.3$ (range $1-17$ ) years and $7.5 \pm 3.5$ (range 2-17) years, respectively (Figure 2).

\section{Agreement between clinical and pathological diagnoses}

Agreement on the diagnosis of NHL (ie, Burkitt's lymphoma and other NHL), other cancer, or chronic noncancerous conditions between clinical diagnoses and pathological diagnoses assigned in Uganda was 91\% (95\% CI 84-95) with a kappa statistic of 0.84 (95\% CI 0.75-0.92; $P<0.001)$. The agreement between clinical diagnoses and pathological diagnoses assigned in The Netherlands was 49\% (95\% CI $40-59)$ with a kappa statistic of $0.04(95 \%$ CI $-0.10-0.17$; $P=0.612$ ). Of the 46 children with a clinical diagnosis of a chronic noncancerous condition, 34 had a cancer diagnosis according to the laboratory in The Netherlands (Table 2).

Further comparison by NHL subtype (ie, Burkitt's lymphoma or other NHL), other cancer, or a chronic noncancerous condition, showed an agreement between clinical diagnoses and pathological diagnoses assigned in Uganda of $69 \%$ (95\% CI 59-77) with a kappa statistic of 0.56 (95\% CI $0.44-0.67 ; P<0.001)$. Agreement on NHL subtypes 


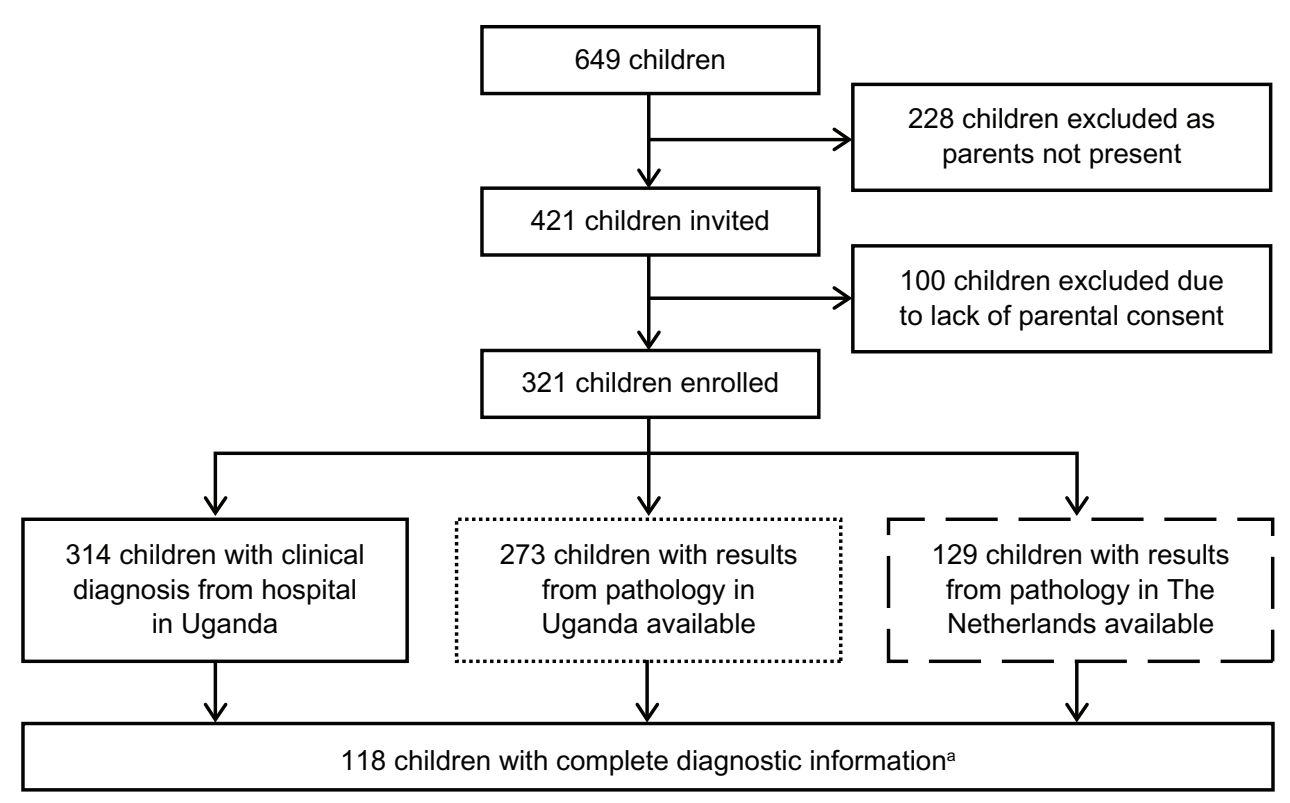

Overlap of the available results from the KNRH and the two pathology laboratories

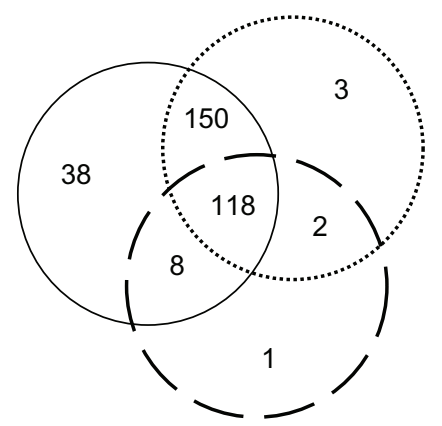

Figure I Flow of data for participants of this study on childhood lymphoma at the Mulago National Referral Hospital with details on overlap between the Mulago National Referral Hospital and the two pathology laboratories.

Note: ${ }^{a}$ Complete diagnostic information includes the clinical diagnosis, the pathological diagnosis assigned in Uganda, and the pathological diagnosis assigned in The Netherlands.

(ie, Burkitt's lymphoma or other NHL), other cancer, and a chronic noncancerous condition between clinical diagnoses and pathological diagnoses assigned in The Netherlands was $32 \%(95 \%$ CI $24-41)$ with a kappa statistic of 0.05 (95\% CI $-0.06-0.16 ; P=0.326$, Table 3). A direct comparison of pathological diagnoses assigned in Uganda and The Netherlands showed an agreement of 36\% (95\% CI 28-46) with a kappa value of $0.11(95 \% \mathrm{CI}-0.01-0.24 ; P=0.0459$, Table 4).

\section{Discussion}

In this study, we compared diagnoses assigned by clinicians in Uganda who initially enrolled children with suspected NHL at the MNRH, and those assigned by pathologists in Uganda and The Netherlands using the same tissue samples. Pathological diagnosis was based on histology using hematoxylin and eosin staining both in Uganda and The Netherlands, whereas diagnoses were further detailed by several additional tests in The Netherlands.
Although agreement between clinical diagnoses and pathological diagnoses assigned in Uganda was relatively high, agreement between clinical diagnoses and pathological diagnoses in The Netherlands was much lower. This discrepancy could be partially explained by the fact that the pathologists in Uganda were not blinded to information on clinical diagnosis, and may therefore have been influenced by the clinical diagnosis when in doubt about the pathological findings. This was unavoidable given that the study was conducted in a routine clinical setting. The pathologists in The Netherlands had no access to clinical diagnoses, and based their diagnosis solely on the biological samples.

The agreement between pathological diagnoses made in Uganda and in The Netherlands was very low (36\%). This weak agreement is probably due to the fact that pathological diagnosis in Uganda is performed at a very basic level, whereas pathologists in The Netherlands have access to additional tests for subsequent detailed characterization by immunohistochemistry, and are thus able to make more 


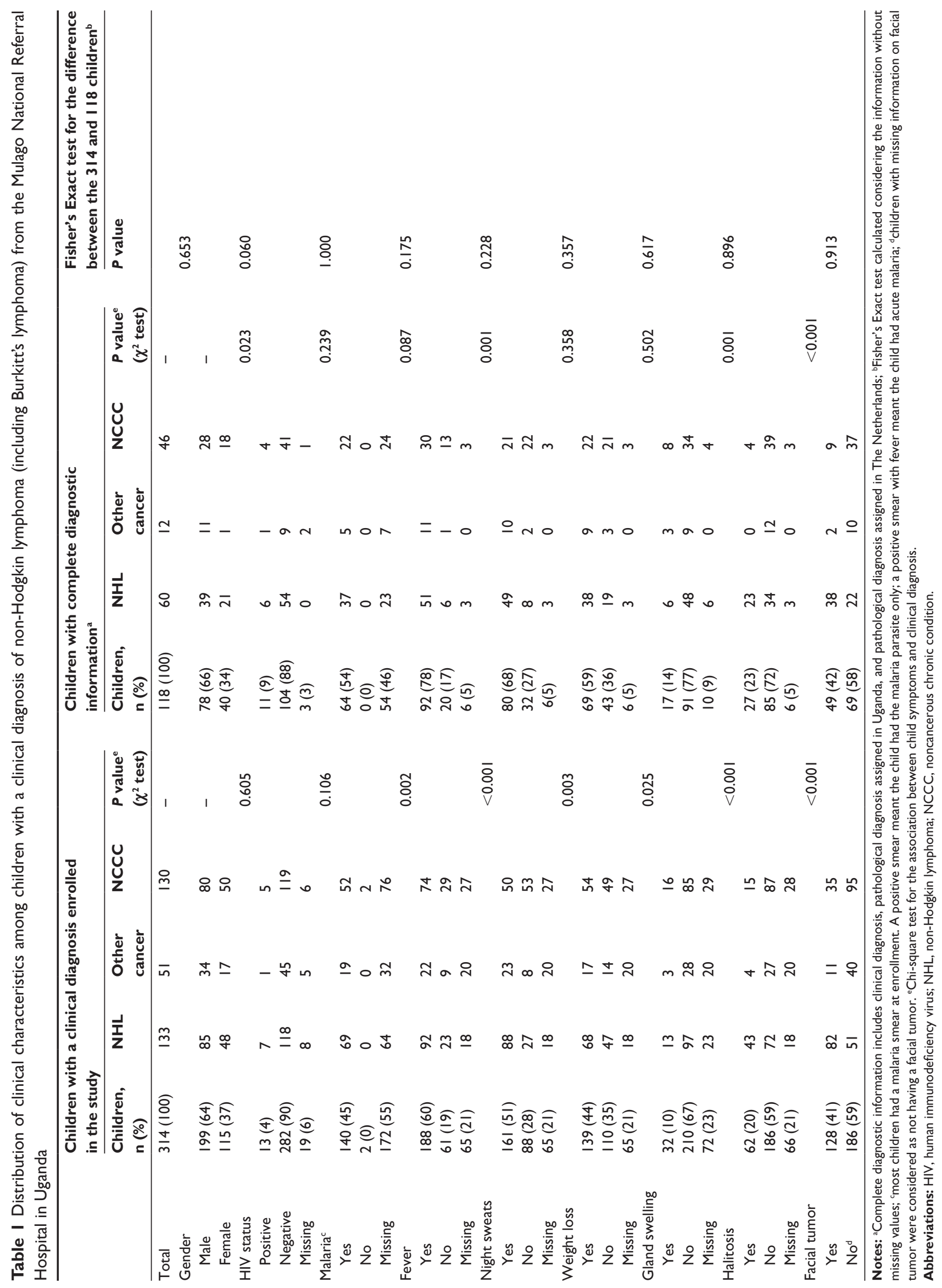



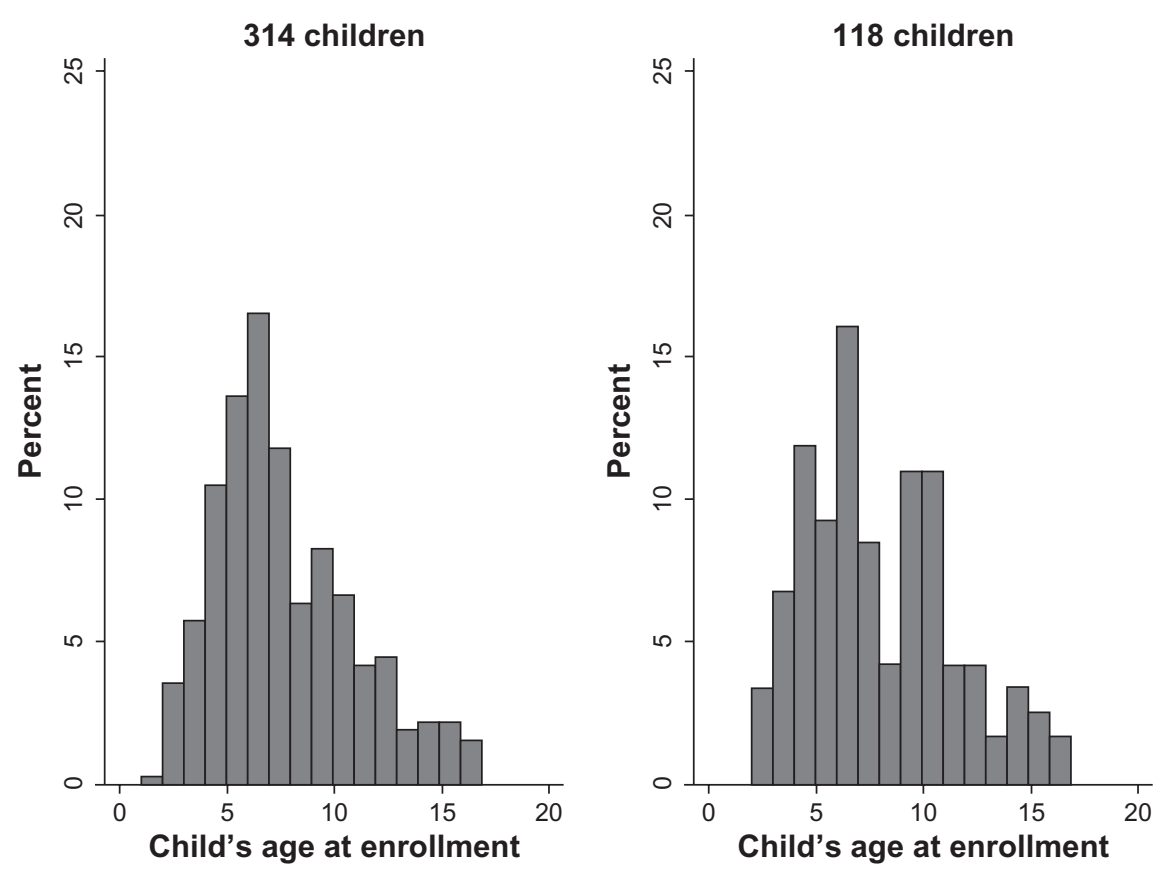

Figure 2 Age distributions of the 314 children (mean age $7.2 \pm 3.3$ [range I-17] years) with a clinical diagnosis at Mulago National Reference Hospital in Uganda and the 118 children (mean age $7.5 \pm 3.5$ [range 2-17] years) with complete diagnostic information. ${ }^{2}$

Note: ${ }^{a}$ Complete diagnostic information includes the clinical diagnosis, the pathological diagnosis assigned in Uganda, and the pathological diagnosis assigned in The Netherlands.

precise diagnoses. Assuming that the diagnoses assigned in The Netherlands are correct, which is a fair assumption given the state-of-the-art laboratory and highly trained staff specialized in the diagnosis of lymphomas, particularly Burkitt's lymphoma, our study indicates that only $52 \%$ of the children with Burkitt's lymphoma, and only $22 \%$ of the children with NHL in Uganda are being correctly diagnosed. Inevitably, inappropriate cancer diagnoses lead to inadequate patient management, consequently compromising the prognosis.

Table 2 Comparison of agreement between clinical diagnoses assigned at the Mulago National Referral Hospital in Uganda for nonHodgkin lymphoma (including Burkitt's lymphoma), other cancer, and noncancerous chronic conditions, and the pathology laboratories in Uganda and The Netherlands, for the 118 children with complete diagnostic information ${ }^{\mathrm{a}}$

\begin{tabular}{|c|c|c|c|c|}
\hline & \multicolumn{4}{|c|}{ Clinical diagnosis from Uganda } \\
\hline & NHL, n (\%) & Other cancer, $\mathbf{n}(\%)$ & NCCC, n (\%) & Total, n (\%) \\
\hline \multicolumn{5}{|l|}{ Pathological diagnosis from Uganda } \\
\hline $\mathrm{NHL}$ & $58(94)$ & $I(2)$ & $3(5)$ & $62(100)$ \\
\hline Other cancer & I (8) & $9(69)$ & $3(23)$ & $13(100)$ \\
\hline NCCC & I (2) & $2(5)$ & $40(93)$ & $43(100)$ \\
\hline Total & $60(5 \mathrm{I})$ & $12(10)$ & $46(39)$ & $118(100)$ \\
\hline Kappa test statistic $(95 \% \mathrm{Cl})$ & & & & $0.84^{b}(0.75,0.92)$ \\
\hline$P$ value of the kappa statistics & & & & $<0.001$ \\
\hline Percentage of agreement $(95 \% \mathrm{Cl})$ & & & & $91 \%(84 \%, 95 \%)$ \\
\hline \multicolumn{5}{|c|}{ Pathological diagnosis from The Netherlands } \\
\hline $\mathrm{NHL}$ & $46(53)$ & $8(9)$ & $33(38)$ & $87(100)$ \\
\hline Other cancer & I (50) & $0(0)$ & $I(50)$ & $2(100)$ \\
\hline NCCC & $13(45)$ & $4(14)$ & $12(4 \mid)$ & $29(100)$ \\
\hline Total & $60(51)$ & $12(10)$ & $46(39)$ & $118(100)$ \\
\hline Kappa test statistic $(95 \% \mathrm{Cl})$ & & & & $0.04(-0.10,0.17)$ \\
\hline$P$ value of the kappa statistics & & & & 0.612 \\
\hline Percentage of agreement $(95 \% \mathrm{Cl})$ & & & & $49 \%(40 \%, 59 \%)$ \\
\hline
\end{tabular}

Notes: ${ }^{a}$ Complete diagnostic information: clinical diagnosis, pathological diagnosis assigned in Uganda and pathological diagnosis assigned in The Netherlands; ${ }^{b}$ we also performed a kappa test result including 268 children with both preliminary clinical diagnosis and pathological diagnosis from Uganda, which yielded a kappa statistic of 0.74 (95\% Cl 0.66-0.8I; $P<0.000 \mathrm{I})$, and a percentage agreement of $84 \%$ (95\% Cl 79-88).

Abbreviations: $\mathrm{Cl}$, confidence interval; NHL, non-Hodgkin lymphoma; NCCC, noncancerous chronic condition; $\mathrm{n}$, number of children. 
Table 3 Comparison of agreement between clinical diagnosis at the Mulago National Referral Hospital in Uganda for Burkitt's lymphoma, other non-Hodgkin lymphoma (excluding Burkitt's lymphoma), other cancer, and noncancerous chronic conditions, and pathology laboratories in Uganda and in The Netherlands, for the II 8 children with complete diagnostic information ${ }^{\mathrm{a}}$

\begin{tabular}{|c|c|c|c|c|c|}
\hline & \multicolumn{4}{|c|}{ Clinical diagnosis from Uganda } & \multirow[t]{2}{*}{ Total, n (\%) } \\
\hline & $\overline{B L}$, n (\%) & Other NHL, n (\%) & Other cancer, n (\%) & NCCC, n (\%) & \\
\hline \multicolumn{6}{|l|}{ Pathological diagnosis from Uganda } \\
\hline BL & $21(62)$ & $10(29)$ & $0(0)$ & $3(9)$ & $34(100)$ \\
\hline Other NHL & $16(57)$ & II (39) & I (4) & $0(09)$ & $28(100)$ \\
\hline Other cancer & $0(0)$ & $I(8)$ & $9(69)$ & $3(239)$ & $13(100)$ \\
\hline $\mathrm{NCCC}$ & $I(2)$ & $0(0)$ & $2(5)$ & 40 (939) & $43(100)$ \\
\hline Total & $38(32)$ & $22(19)$ & $12(10)$ & $46(399)$ & $118(100)$ \\
\hline Kappa test statistic $(95 \% \mathrm{Cl})$ & & & & & $0.56(0.44-0.67)$ \\
\hline$P$ value of the kappa statistics & & & & & $<0.0001$ \\
\hline Percentage of agreement $(95 \% \mathrm{Cl})$ & & & & & $69 \%(59 \%, 77 \%)$ \\
\hline \multicolumn{6}{|c|}{ Pathological diagnosis from The Netherlands } \\
\hline BL & $19(45)$ & $10(24)$ & $0(0)$ & $13(3 \mid)$ & $42(100)$ \\
\hline Other NHL & $10(22)$ & $7(16)$ & $8(18)$ & $20(44)$ & $45(100)$ \\
\hline Other cancer & I (50) & $0(0)$ & $0(0)$ & $\mathrm{I}(50)$ & $2(100)$ \\
\hline $\mathrm{NCCC}$ & $8(28)$ & $5(17)$ & $4(14)$ & $12(4 \mid)$ & $29(100)$ \\
\hline Total & $38(32)$ & $22(19)$ & $12(10)$ & $46(39)$ & $118(100)$ \\
\hline Kappa test statistic $(95 \% \mathrm{Cl})$ & & & & & $0.05(-0.06,0.16)$ \\
\hline$P$ value of kappa statistics & & & & & 0.326 \\
\hline Percentage of agreement $(95 \% \mathrm{Cl})$ & & & & & $32 \%(24 \%, 41 \%)$ \\
\hline
\end{tabular}

Notes: a Complete diagnostic information: clinical diagnosis, pathological diagnosis assigned in Uganda, and pathological diagnosis assigned in The Netherlands; ${ }^{\mathrm{C}}$ combination of variable clinical diagnosis of NHL from Uganda used in Table 2 and "assessment of facial tumor" ("yes" if patient had facial tumor, and "no" otherwise). A patient here is classified as $\mathrm{BL}$ if she or he had both a clinical diagnosis of $\mathrm{NHL}$ and a facial tumor.

Abbreviations: BL, Burkitt's lymphoma; NHL, non-Hodgkin lymphoma; NCCC, noncancerous chronic condition; n, number of children.

The strengths of this study include the study setting, namely a national cancer treatment center in Kampala and its affiliate pathology laboratory, the relatively large sample size, and the fact that the pathological diagnoses were based on the same tissue samples in Uganda and in The Netherlands. Moreover, we were able to compare clinical diagnoses in Uganda with histological diagnoses assigned in Uganda and in The Netherlands.

The main weaknesses of the study are that a large number of patients had to be excluded from the final analysis for diverse reasons, and that many tissue samples could not be properly analyzed in The Netherlands for technical reasons, including poor tissue processing or storage problems back in Uganda. Our analysis comparing the 314 children enrolled in the study with a clinical diagnosis and the 118 children with complete diagnostic information did not reveal significant differences in terms of age distribution, prevalence of human immunodeficiency virus, or symptoms associated with their medical conditions. This is quite reassuring, and suggests that no systematic exclusion of a particular group of children from the final analysis took place. Still, it could be correctly argued that the children included in our study are not a truly

Table 4 Comparison of agreement between the pathological diagnoses assigned in Uganda and The Netherlands, for the II8 children with complete diagnostic information ${ }^{\mathrm{a}}$

\begin{tabular}{|c|c|c|c|c|c|}
\hline & \multicolumn{4}{|c|}{ Pathological diagnosis from Uganda } & \multirow[t]{2}{*}{ Total, n (\%) } \\
\hline & BL, n (\%) & Other NHL, n (\%) & Other cancer, n (\%) & NCCC, n (\%) & \\
\hline \multicolumn{6}{|c|}{ Pathological diagnosis from The Netherlands } \\
\hline BL & $22(52)$ & $7(17)$ & I (2) & $12(29)$ & $42(100)$ \\
\hline Other NHL & $8(18)$ & $10(22)$ & $8(18)$ & $19(42)$ & $45(100)$ \\
\hline Other cancer & $0(0)$ & $\mathrm{I}(50)$ & $0(0)$ & I (0) & $2(100)$ \\
\hline $\mathrm{NCCC}$ & $4(14)$ & $10(34)$ & $4(14)$ & $11(38)$ & $29(100)$ \\
\hline Total & $34(29)$ & $28(24)$ & $13(11)$ & $43(36)$ & $118(100)$ \\
\hline Kappa test statistic $(95 \% \mathrm{Cl})$ & & & & & $0.11(-0.01,0.24)$ \\
\hline$P$ value of kappa statistics & & & & & 0.0459 \\
\hline Percentage of agreement $(95 \% \mathrm{Cl})$ & & & & & $36 \%(28 \%, 46 \%)$ \\
\hline
\end{tabular}

Note: a Complete diagnostic information: clinical diagnosis, pathological diagnosis assigned in Uganda and pathological diagnosis assigned in The Netherlands.

Abbreviations: BL, Burkitt's lymphoma; Cl, confidence interval; NHL, non-Hodgkin lymphoma; NCCC, noncancerous chronic condition; n, number of children. 
representative sample of all children with NHL in Uganda, because only the children who actually reached the Uganda Cancer Institute (the only specialized cancer hospital in the country), had informed consent from a parent or guardian, and in addition had a biological sample collected and analyzed, were included. However, given that the aim of the study was to verify the agreement between clinical and pathological diagnoses within the hospital population, we could only include children with complete diagnostic information in the final analyses. Moreover, conclusions were drawn based on internal comparisons within the study population only, thus internal validity was assured. Although only 118 children had complete diagnostic information, a larger number of samples would not necessarily have increased the probability of agreement across the three diagnostic procedures.

Unfortunately, our findings in Uganda may not be unique. A recent survey of infrastructure and capacity for diagnosis in Africa indicated an unacceptably low standard of histopathology, probably due to the lack of advanced technology and shortage of pathologists. ${ }^{18}$ It has been suggested that the solution to the poor quality of diagnosis in low-income and middle-income countries is to form partnerships with centers of excellence in high-income countries, where samples can be submitted or data and images shared, in particular when rapid and high-quality Internet-based digital imaging is involved. There is merit to this suggestion, but the downside is that it may not improve primary tissue processing expertise, and may neglect local capacity building and sustainability. Moreover, without a strong infrastructure for obtaining and processing good quality samples, the quality of shipped slides and samples will still affect the quality of diagnosis. ${ }^{19}$ A study from Ghana is instructive in this regard. It evaluated the feasibility of using a UK-based diagnostic laboratory to improve the diagnosis and management of lymphoproliferative disorders. ${ }^{9}$ Although refined diagnoses were achieved, the implementation was challenging in Ghana due to lack of local capacity and specialized facilities to produce adequate samples. Therefore, this study confirms the need for a secure local structure that can obtain and process samples, and make the initial diagnosis.

The present report provides evidence of a need to improve the quality of diagnosis of childhood NHL in Uganda. There is a clear need to create an efficient system within the public health sector for collecting, handling, processing, and storing biological samples. Moreover, there is a need for a national reference laboratory specialized in cancer that can render dependable clinical histopathology diagnoses.
This laboratory should be adequately equipped and staffed, have reliable access to laboratory supplies, clinical guidelines, standard operating procedures, algorithms for diagnosis, and regular quality control of all procedures. Such a laboratory should be an integral part of the public health system in Uganda, and needs to be affordable or free of charge for low-income families with children having a suspected cancer diagnosis. It could also serve as a national center for continued medical education and training for pathologists and clinicians. ${ }^{20}$ In addition, more advanced skills and technology for pathological diagnosis, such as basic immunohistochemical analysis, should be introduced whenever feasible in tertiary hospitals around the country. Clinicians should also be trained to provide early leads to pathologists about the possible diagnosis, which brings the added advantage of improved teamwork. Improvements in the capacity for tissue handling, processing, storage, and pathology would also expand the clinical and pathological research opportunities for the country, because it would make available a set of reliable biological samples. This would be of particular relevance for research on childhood NHL, especially Burkitt's lymphoma, given that Uganda is one of the countries with the highest number of incident cases of these diseases.

\section{Conclusion}

Clinical diagnosis alone should not be the basis for initiating treatment of any type of NHL in Uganda, including Burkitt's lymphoma, because the probability of diagnostic error is high. A high-quality national histopathology diagnosis laboratory specialized in cancer is warranted in Uganda. Clinical diagnosis of NHL in Uganda has a high probability of error compared with both pathological diagnosis in Uganda and in The Netherlands. The agreement between pathological diagnosis for NHL in Uganda and The Netherlands is very low.

\section{Acknowledgments}

The authors would like to thank the children who participated in this study and their families; Fred Okuku, David Sentongo, Laurence Olweny, and Irene Judith Nasozi for study support; and Yusuf Mulumba and Pouran Almstedt for data management; Tineke Vendrig for detailed EBER and IHC tissue analysis; and Trudy Perdrix-Thoma for editorial assistance and language review. This study was financed by a grant from the Swedish Agency for Help to Development for a research collaboration between Makerere University and the Karolinska Institutet, Stockholm, Sweden. 


\section{Disclosure}

The authors report no conflicts of interest in this work.

\section{References}

1. Tumwine LK, Agostinelli C, Campidelli C, et al. Immunohistochemical and other prognostic factors in B cell non Hodgkin lymphoma patients, Kampala, Uganda. BMC Clin Pathol. 2009;9:11.

2. Perkins SL. Work-up and diagnosis of pediatric non-Hodgkin's lymphomas. Pediatr Dev Pathol. 2000;3:374-390.

3. Cairo MS, Raetz E, Lim MS, Davenport V, Perkins SL. Childhood and adolescent non-Hodgkin lymphoma: new insights in biology and critical challenges for the future. Pediatr Blood Cancer. 2005;45:753-769.

4. Kalungi S, Wabinga H, Molven A, Bostad L. Lymphomas diagnosed in Uganda during the HIV/AIDS pandemic. East Afr Med J. 2009;86:226-232.

5. Frost M, Newell J, Lones MA, Tripp SR, Cairo MS, Perkins SL. Comparative immunohistochemical analysis of pediatric Burkitt lymphoma and diffuse large B-cell lymphoma. Am J Clin Pathol. 2004;121:384-392.

6. Cairo MS, Sposto R, Perkins SL, et al. Burkitt's and Burkitt-like lymphoma in children and adolescents: a review of the Children's Cancer Group experience. Br J Haematol. 2003;120:660-670.

7. Harris NL, Jaffe ES, Diebold J, Flandrin G, Muller-Hermelink HK, Vardiman J. Lymphoma classification - from controversy to consensus: the R.E.A.L. and WHO classification of lymphoid neoplasms. Ann Oncol. 2000;11 Suppl 1:3-10.

8. Ogwang MD, Zhao W, Ayers LW, Mbulaiteye SM. Accuracy of Burkitt lymphoma diagnosis in constrained pathology settings: importance to epidemiology. Arch Pathol Lab Med. 2011;135:445-450.

9. Parkins E, Owen RG, Bedu-Addo G, et al. UK-based real-time lymphoproliferative disorder diagnostic service to improve the management of patients in Ghana. J Hematop. 2009;2:143-149.

10. Naresh KN, Ibrahim HA, Lazzi S, et al. Diagnosis of Burkitt lymphoma using an algorithmic approach - applicable in both resource-poor and resource-rich countries. Br J Haematol. 2011;154: $770-776$.
11. Stefan DC, Siemonsma F. Delay and causes of delay in the diagnosis of childhood cancer in Africa. Pediatr Blood Cancer. 2011;56: 80-85.

12. Orem J, Mulumba Y, Algeri S, et al. Clinical characteristics, treatment and outcome of childhood Burkitt's lymphoma at the Uganda Cancer Institute. Trans R Soc Trop Med Hyg. 2011;105:717-726.

13. Tumwine LK, Campidelli C, Righi S, Neda S, Byarugaba W, Pileri SA. B-cell non-Hodgkin lymphomas in Uganda: an immunohistochemical appraisal on tissue microarray. Hum Pathol. 2008;39:817-823.

14. Lukande R, Wabinga HR, Tumwine LK. Burkitt's lymphoma in Uganda: the role of immunohistochemistry in diagnosis. East Afr Med J. 2008;85:207-212.

15. Lassmann H, Niedobitek G, Aloisi F, Middeldorp JM. Epstein-Barr virus in the multiple sclerosis brain: a controversial issue - report on a focused workshop held in the Centre for Brain Research of the Medical University of Vienna, Austria. Brain. 2011;134:2772-2786.

16. Clopper CJ, Pearson ES. The use of confidence or fiducial limits illustrated in the case of the binomial. Biometrika. 1934;26: 404-413.

17. Fleiss JL, Levin B, Paik MC. Statistical Methods for Rates and Proportions. 3rd ed. Hoboken, NJ: John Wiley and Sons; 2003.

18. Naresh KN, Raphael M, Ayers L, et al. Lymphomas in sub-Saharan Africa - what can we learn and how can we help in improving diagnosis, managing patients and fostering translational research? Br J Haematol. 2011;154:696-703.

19. Mbulaiteye SM, Talisuna AO, Ogwang MD, McKenzie FE, Ziegler JL, Parkin DM. African Burkitt's lymphoma: could collaboration with HIV-1 and malaria programmes reduce the high mortality rate? Lancet. 2010;375:1661-1663.

20. Proctor IE, McNamara C, Rodriguez-Justo M, Isaacson PG, Ramsay A. Importance of expert central review in the diagnosis of lymphoid malignancies in a regional cancer network. J Clin Oncol. 2011;29: 1431-1435.
Clinical Epidemiology

\section{Publish your work in this journal}

Clinical Epidemiology is an international, peer-reviewed, open access journal focusing on disease and drug epidemiology, identification of risk factors and screening procedures to develop optimal preventative initiatives and programs. Specific topics include: diagnosis, prognosis, treatment, screening, prevention, risk factor modification, systematic

Submit your manuscript here: http://www.dovepress.com/clinical-epidemiology-journal

\section{Dovepress}

reviews, risk \& safety of medical interventions, epidemiology \& biostatical methods, evaluation of guidelines, translational medicine, health policies \& economic evaluations. The manuscript management system is completely online and includes a very quick and fair peer-review system, which is all easy to use. 